\title{
Applications of a computer algebra system for teaching bivariate relationships in statistics courses
}

\author{
FRANK HASSEBROCK and RITA SNYDER \\ Denison University, Granville, Ohio
}

\begin{abstract}
Maple, a computer algebra system, is used in undergraduate psychology courses to promote students' conceptual learning of basic principles associated with bivariate relationships. Maple's symbolic computation, graphic displays, and animation capabilities are used along with other classroom activities to study concepts related to (1) correlation coefficients, scatterplots, and regression lines, (2) factors that affect the magnitude of sample correlations, (3) inferential tests, and (4) prediction error. For each of the 15 Maple procedures, students select population correlation values and examine the effects of different values on computer-generated graphical representations of scatterplots, regression lines, and sample correlations. The procedures are described and examples of exercises to support their use are provided.
\end{abstract}

Psychology instructors make frequent use of computers in teaching statistics courses, and statistical computation software can help students learn procedural skills in data analysis and statistical interpretation (Oswald, 1996). Conceptual understanding of statistical principles can be enhanced by the unique pedagogical features available from computer algebra systems (CAS) such as Maple. CASs provide an integrated set of symbolic, numeric, and visual representations that support students' reasoning rather than only procedural computation skills (Karian \& Tanis, 1995). For example, Snyder (1995) developed and used a set of 20 Maple procedures in an introductory statistics course for undergraduate psychology students to promote conceptual learning of principles for sampling distributions, statistical decision making, error, and power. This paper describes a new set of 15 Maple procedures which students use to examine statistical principles associated with bivariate relationships, including (1) correlation coefficients, scatterplots, and regression lines, (2) factors that affect the magnitude of sample correlation coefficients, (3) inferential tests, and (4) prediction error.

The use of computers to help students understand correlation topics has focused on computer-displayed graphical presentations, especially scatterplots (e.g., Goldstein

This work was supported in part by Grant P116B30079 from the Fund for the Improvement of PostSecondary Education (FIPSE) of the Department of Education. The procedures run with both Macintosh and DOS versions of Maple. Contact ${ }^{\circ}$ Waterloo Maple Software at info@ maplesoft.on.ca, or at (800) 267-6583, for specific information about computer requirements for each version. Additional software needed to run the statistical procedures described in this paper will be provided by the authors on either a Macintosh or a DOS diskette. Contact F. Hassebrock or R. Snyder. Department of Psychology, Denison University, Granville, OH 43023 (e-mail: hassebrock@denison.edu or snyder (a)denison.edu).
\& Strube, 1995; Meyer \& Shinar, 1992). In extending our earlier work with Maple to the new set of procedures described below, we also sought to enhance our students' conceptual understanding of bivariate relationships through visual and graphical representations (many involving scatterplots and regression lines) without direct use of mathematical computations or discrete sets of data. The integration of symbolic computation, graphical displays, and animation capabilities afforded by these procedures is intended to promote students' active learning of statistical concepts (Jackson, Edwards, \& Berger, 1993).

The procedures can be used on either a Macintosh or a DOS-based system with Maple software installed. However, neither students nor instructors have to learn to program with Maple; rather, they simply enter procedure names (a single command produces a brief on-screen description of the procedures). Instructors can develop learning exercises for these procedures (as illustrated in the examples we provide below) and incorporate them into either classroom work or assignments with a small amount of development effort and operating instruction. The procedures also have several user-friendly features. For example, on-screen prompts are provided for each value students must select as input. As each procedure is being executed, a brief description of the statistical principle illustrated or content of the output is displayed on the screen. Colored graphical representations of bivariate scatterplots and regression lines are presented with many of the procedures, and all of the procedures' graphs are labeled with relevant parameter or statistic values. Informative error traps are built in; for example, students are reminded that values of correlation coefficients must be between -1.0 and +1.0 if they select an inappropriate input value. The similarity of the graphical displays across the set of topics explored with these procedures encourages students to recognize conceptual linkages 
while exploring the underlying statistical principles of bivariate relationships. The Appendix lists the 15 procedures and their basic operation. The four instructional areas for examining bivariate relationships that can be explored with these procedures, as well as examples of learning exercises we developed for these procedures, are described in the next sections.

\section{Correlation Coefficients, Scatterplots, and Regression Lines}

A set of six procedures allows students to examine relationships among correlation coefficients, scatterplots, and regression lines. For three of these procedures, students select a population correlation value $(r h o)$ and the procedure produces a graphical representation of data for a random sample from that population. Each time a procedure is executed, a new random sample is obtained. Animations of each of these procedures are also included in this group and are based on independent random samples from populations with incrementally increasing (or decreasing) values of $r h o$.

An illustration of how these procedures are used to introduce the concept of correlation to students in an undergraduate research methods class is provided by the following exercise. We begin with a lecture on different correlational research methods and the type of research conclusions that can be reached from a bivariate relationship. A correlational research problem is presented, and then a scatterplot is constructed on the blackboard and its essential features are described. Then we ask students to envision mentally how the scatterplot would change in shape as the magnitude and direction of the correlation changes for the two variables in the problem. At this point, we distribute an assignment and ask students to work in pairs at the Macintosh terminals located in our statistics lab. The assignment has a general question, "How does a scatterplot represent a sample correlation?" and the students are asked to answer the following questions by using the scatter procedure.

1. Select a value for rho representing a weak correlation. What features of the scatterplot illustrate the weak relationship?

2. Select a value for rho representing a strong correlation.

3. Compare the scatterplots generated for 1 and 2 . In what ways are they different?

4. How do the values for $r$ on your scatterplots compare with the values of rho you defined? If they are different in one or both cases, why are they different? Why might they be the same sometimes?

Students answer these questions after they use scatter to select several different population correlation values and compare the scatterplots of data for samples from these populations. Then, the animated version of this procedure (animscatter) is shown to the students in order to provide a dynamic representation of a scatterplot which changes as the correlation value varies between -1.0 and +1.0 . Thereafter, the introduction of regline and scatreg (and their animated versions) asks students to consider how prediction and prediction errors depend upon the magnitude of the correlation as shown by the spread of data points around the plotted regression line. During this exercise, students are encouraged to select a variety of population values in order to explore the relationship between the shape of the scatterplot and the slope of the regression line.

The Maple-based exercises provide students with a computer-based conceptual introduction to the relationship among correlation coefficients, scatterplots, and regression lines. Since it is important for students to develop procedural skills for computing and interpreting statistics, use of a statistical software package (SPSS) is also emphasized in our research methods and statistics courses. For example, after the conceptual introduction provided by the Maple procedures, students are given a research problem with an accompanying data set and are provided with instructions for computing the correlation coefficient by hand and by using SPSS. Interpretation of the calculated correlation value in the context of the research problem can be discussed in terms of the student's conceptual understanding of correlation magnitude as well as appropriate applications of that understanding for the description of behavior.

\section{Factors Affecting the Magnitude of Correlation Coefficients}

The second group of five procedures is used by students to examine factors that may or may not affect the magnitude of $r$ for a random sample: nonlinearity of the relationship, range-restriction of one variable, and sample size. For example, to help students appreciate the impact of range-restriction on reducing the magnitude of the calculated value of $r$, the procedure restrict is employed in a homework assignment titled, "How does rangerestriction affect the value of $r$ ?" The assignment consists of the following:

The restrict procedure obtains a large sample (minimum $N=100$ ) from a population with a rho value you specify and displays the standard deviations of both variables as well as $r$. After you restrict the range of the $X$ variable, the standard deviation and $r$ are recomputed for the "new" restricted subsample. In addition, scatterplots of the full sample and the restricted subsample are displayed for the comparison. Select a value of $r$ ho that represents a strong correlation and set $N$ to 100 . You may restrict the range to any portion of the range you wish. Be restrictive: $10 \%$ of the range is not too restrictive.

1. Compare the standard deviations of the values of $X$ and $Y$ for the full sample and the restricted subsample. Why do they differ?

2. Compare the two scatterplots ("pull" the top one over on your screen so you can examine them simultaneously). In what ways do they differ?

3. Compare the values of $r$ for the full sample and for the restricted subsample (shown on the scatterplots). Why do they differ?

As students use restrict to answer these questions, scatterplots of the original sample and the sample with rangerestriction are produced on identically scaled axes. By 
comparing the values of $r$ and the standard deviation of the original data set with that of the restricted data set, students are able to articulate the effect of range-restriction on limiting their success in identifying the actual magnitude of the correlation. After students have tested different values of $r$ and restricted at least two different parts of the range of $X$, the animated procedure (animrestrict) is executed, and then a class discussion allows students to share their outcomes and confirm the generality of the principles across a variety of correlation coefficient values and range-restriction options.

The other procedures in this set address additional topics typically presented in statistics courses. For example, the procedure animnonlin is used to help demonstrate that strong relationships may be nonlinear, but the correlation coefficients the students are learning to use do not index the strength of these relationships. The procedure displays the value of Pearson's $r$ for nonlinear functions: as nonlinearity increases, the value of $r$ decreases. Students are encouraged to recall nonlinear relationships from previous coursework (e.g., motivation research) and to recognize the importance of examining visual displays of relationships as part of determining whether the use of Pearson's $r$ will provide an accurate description. Another factor students explore is the effect of sample size on the magnitude of $r$. The procedure $n$ corr permits students to select a variety of sample sizes and note the effect (or lack of an effect) on the value of $r$. By comparing the values of $r$ resulting from several random samples of the same size with the values of $r$ resulting from several random samples of differing sizes from the same population, students discover that variations in the magnitude of $r$ are attributable to sampling error rather than to sample size.

\section{Correlation and Inferential Tests}

A pair of procedures (critcorr and ncritcorr) was created to link descriptive and inferential applications. Critcorr generates a plot of the critical value of $r$ as a function of sample size. The second procedure, ncritcorr, helps illustrate the different effects of increasing sample size on parameter estimation and statistical power. Based on a student-selected rho value, this procedure obtains five random samples for three different sample sizes $(n=5,15$, and 25) and plots the values of $r$ for each sample and the critical value of $r$ for each $n$. The graphical display produced by this procedure shows that the average value of $r$ s for each sample size is approximately equal to $r h o$; by contrast, most values of $r$ fall below the critical value for tests with the smaller samples but above the critical value for tests with the largest sample, demonstrating how sample size affects the power of inferential tests of the null hypothesis $r h o=0$.

\section{Correlation and Prediction Error}

The final pair of procedures focuses on prediction error. One procedure ( prederror) permits students to define two variables by their respective means and standard deviations and specify the value of rho. Based on this input, a graph showing the scatterplot, regression line, and standard error of the estimate for a random sample from the population is produced. There also is an animated version (animprederror) of the procedure.

\section{Conclusion}

These procedures take advantage of the computing and graphical capabilities of a CAS such as Maple; however, to be used effectively, the procedures are embedded within instructional contexts like the examples we have provided in this paper. Assessment of how the procedures (and the set developed earlier by Snyder, 1995) have impacted our students' learning has been primarily accomplished by examining (and grading) students' responses to various types of classroom exercises and homework assignments (like the ones we have described in this paper) and responses to short-answer and essay questions on exams. Thus, our assessment to date has been more formative than summative. We also examined students' comments from course evaluations. For example, in an upper-level statistics course, 22 students responded to open-ended questions and rating-scale questions included in a course evaluation form. The majority of students (73\%) thought that the procedures' graphs enhanced their conceptual understanding through visualization and mental imagery. Only 2 students claimed that the procedures were not helpful. Although only 2 students had had prior experience with Maple, the group's mean self-rating of competence in using the procedures to complete homework assignments was $4.5(S D=1.4)$ on a 7 -point scale $(1=$ very poor and $7=$ very good $)$. The mean self-rating of "frustration level" in using the procedures was $3.4(S D=1.7)(1=$ not at all frustrated and $7=$ very frustrated $)$.

The Maple-based procedures described in this paper provide educational support different from that provided by statistical software packages like SPSS. Although use of these packages also improves students' ability to employ statistical analyses in a research context, these packages are not as useful for generating the dynamic visual displays (animations of statistical relationships) that support better understanding of the statistical concepts being applied. Also, because packages like SPSS depend primarily on specific data sets for their operation, manipulation of population characteristics and examination of consequent changes in sample characteristics are not easily accomplished. Similarly, the ability to use repeated sampling to examine error associated with estimation of population characteristics is very limited. To the extent that students' ability to use statistics effectively is improved by their developing conceptual as well as procedural knowledge, the implementation of Maplebased procedures like those described here in undergraduate statistics courses will prove beneficial.

We have used the Maple procedures and learning exercises to provide a conceptual focus to complement the procedural and interpretive skills students also acquire 
by using computational software such as SPSS. Our students do learn the intricacies of SPSS in order to build and manipulate data files and to execute a variety of statistical procedures, whereas they do not have to learn the Maple commands because the procedures they use are completely scripted. By having students explore multiple and alternative computer-based representations of bivariate relationships, an attempt has been made to enhance problem-solving skills and understanding in a well-designed computing environment (Jackson et al., 1993; Sherwood, Kinzer, Bransford, \& Franks, 1987).

\section{REFERENCES}

GoldSTEIN, M. D., \& STRUBE, M. J. (1995). Understanding correlations: Two computer exercises. Teaching of Psychology, 22, 205-206.

JaCKSON, D. F., EDWARdS, B. J., \& Berger, C. F. (1993). Teaching the design and interpretation of graphs through computer-aided graphical data analysis. Journal of Research in Science Teaching, 30, 483-501.

Karian, Z. A., \& TANis, E. A. (1995). Probability and statistics explorations with Maple. Englewood Cliffs, NJ: Prentice-Hall.

MEYER, J., \& SHINAR, D. (1992). Estimating correlations from scatterplots. Human Factors, 34, 335-349.

OsWALD, P. A. (1996). Classroom use of the personal computer to teach statistics. Teaching of Psychology, 23, 124-126.

Sherwood, R. D., Kinzer, C. K., Bransford, J. D., \& Franks, J. J. (1987). Some benefits of creating macro-contexts for science instruction: Initial findings. Journal of Research in Science Teaching, 24, 417-435.

SNYDER, R. (1995). Applications of a computer algebra system in introductory statistics. Behavior Research Methods, Instruments, \& Computers, 27, 139-143.

\section{APPENDIX \\ Maple Procedures for Studying Bivariate Relationships}

The 15 Maple procedures and their basic operation are described for four sets of instructional topics covered in introducing statistical concepts of bivariate relationships. Each procedure provides prompts for all student-selected values.

\section{Relationships Among Correlation Coefficients, Scatterplots, and Regression Lines}

1. scatter. A scatterplot is shown for the correlation coefficient, $r$, of a sample selected from a population with the userspecified rho.

2. animscatter. An animated series of scatterplots is shown as $r$ changes in magnitude and direction from a perfect positive to a perfect negative correlation.
3. regline. The least squares regression line and $r$ for a random sample from a population with a user-specified rho are presented.

4. animregline. An animated series of regression lines is graphed as $r$ increases of decreases in magnitude and changes in direction.

5. scatreg. A scatterplot, regression line, and $r$ are displayed for a random sample from a population with user-specified rho.

6. animscatreg. This procedure animates scatreg.

\section{Factors Affecting the Magnitude of \\ Correlation Coefficients}

7. animnonlin. Changes in $r$ are projected as the relationship between two variables becomes progressively nonlinear, as shown through an animated series of scatterplots.

8. restrict. Students may select a percentage of lower, middle, or upper values from the original sample, and the procedure presents the scatterplot and $r$ for the restriction.

9. animrestrict. This procedure provides an animation of $r e-$ strict.

10. ncorr. Students choose a variety of sample sizes randomly selected from a given population and note the (lack of) effect of changing $n$ on the value of $r$.

11. animncorr. This procedure animates the outcome of changing $n$.

\section{Inferential Tests}

12. critcorr. This procedure generates a plot of the critical value of $r$ as a function of sample size (based on the means and standard deviations chosen by the student and a rho value).

13. ncritcorr. Based on a student-selected rho value, this procedure plots the values of $r$ for five random samples from three different sample sizes ( $n=5,15$, and 25). The critical value of $r$ for each sample size is also shown.

\section{Prediction Error}

14. prederror. Students define two variables by their respective means and standard deviations and specify the value of $r$ ho. Based on this input, a graph showing the scatterplot, regression line, and standard error of the estimate for a random sample from the population is produced.

15. animprederror. This procedure animates the change in standard error of the estimate as the value of $r$ changes from -1.0 to +1.0 .

(Manuscript received September 30, 1996; revision accepted for publication December 11, 1996.) 